\title{
LE COCKTAIL ou de la guerre dans les affaires
}

\author{
Jean-Luc Moriceau
}

«Pourriez-vous me raconter ce qui s'est passé ce soir là ?»

Je tenais enfin un témoin. Depuis que j'avais entendu parlé de ce cocktail secret, ma curiosité avait été aiguisée. Mais durant cinq années, je n'avais rien réussi à apprendre au-delà des rumeurs et des légendes dont cette soirée était entourée. Je devais faire quelques jours plus tard une conférence sur la stratégie d'entreprise et si je pouvais parler de ce secret bien mystérieux, cela allait être assurément un succès.

C'était une femme d'une cinquantaine d'années. Je l'appelai L. Elle ne m'avait pas regardé en face depuis le début. Elle avait refusé que j'enregistre l'entretien. Elle regardait droit devant elle, d'un air froid et déterminé, il y avait quelque chose de cassé dans son attitude. D'un ton un peu agacé, elle lâcha :

« Je ne me souviens plus très bien. Vous savez, ça fait une bonne dizaine d'année aujourd'hui et j'avais beaucoup à faire. Je remplaçais l'assistante du directeur qui était partie en mission, je faisais le service. C'était deux jours après la signature du contrat. C'était le contrat sur lequel ils travaillaient depuis deux ans et demi, qui devait leur permettre de dépasser définitivement leur principal concurrent. Ils semblaient si heureux, si fiers surtout. Il fallait les voir bomber du torse, et s'envoyer des congratulations les uns aux autres.

Ce soir là, un cocktail pour fêter l'événement réunissait les douze principaux dirigeants et deux ou trois hommes que le directeur affectionnait, mais comme à l'habitude ils ne furent que cinq à avoir le droit de parler. Ce soir là c'était la fête et le champagne était de rigueur. Tout le monde se tenait droit, mais l'alcool rendait la situation plus détendue qu'à l'ordinaire.

Vers le milieu de la soirée, le directeur déclara très fort que tous ici étaient passés maitres dans l'art de la guerre et il décida de porter un toast à Polémos. Ce que tout le monde fit. Puis, à la surprise générale, comme à un rite d'initiation, il demanda à chacun du petit groupe qui l'entourait de faire l'éloge de Polémos, sur le modèle du banquet de Platon. Ils rirent tous et soulignèrent que c'était une excellente idée. Vous pensez, c'était une idée du directeur ! Il désigna la personne à sa droite et un par un ils devaient tous faire un discours à la gloire de Polémos.

- Vous rappelez-vous de ce que chacun déclara ? Qui commença à parler ?

L: Je vous l'ai dit, c'est un peu ancien... Pourtant, une soirée comme celle-là, c'est difficile de l'oublier.

- Si vous vous concentrez sur chacun des discours, vous croyez que vous pourriez me les raconter ?

L : Je vais essayer, car il faut que ce soit raconté. Oui, il me faut le raconter à quelqu'un. »

\section{EVE LEFEDRE-SMITH}

«La première à parler était Ève Smith, la directrice des ressources humaines. Elle avait une robe noire moulante, avec une broche brillante figurant un caractère chinois. Ce n'était pas une marante, mais je crois que le directeur l'estimait beaucoup. Elle n'hésita pas une seconde. Sa voix 
grave avait un ton assuré, entrecoupée de petits rires parfois. Je crois pouvoir redire à peu près ce qu'elle a déclaré :

E. Smith : Polémos est le plus puissant et le plus ancien de tous nos guides. Comme le disait déjà en son temps le sage Héraclite, Polémos est père de toute chose. Et j'ajouterai surtout des meilleures choses. Car ce qui rend une femme, un homme, une entreprise capables de se transformer pour arriver au plus haut, pour arriver au meilleur d'eux-mêmes, n'est-ce pas la guerre économique, les conflits et la lutte pour survivre?

Je confie à Polémos ce qu'il y a de plus important. Lorsque je laisse entendre à cinq collaborateurs qu'un poste supérieur sera disponible dans une année, alors je les vois se battre contre eux-mêmes pour se surpasser et surtout surpasser les autres. J'obtiens alors pendant un an la plus grande efficacité, une efficacité bien plus haute que si je leur disais que je les aimais. De même, si je ne laisse que bien peu de chance à l'un d'eux, qu'il est acculé, qu'il sait qu'il ne pourra s'en sortir qu'au prix des plus grands efforts et exploits, ne croyez-vous pas que c'est là qu'il déploiera la plus grande énergie ? C'est bien parce que Polémos est le plus puissant des aiguillons. Et qu'il est, dans les affaires des hommes, celui qui pousse la ressource humaine à ses plus remarquables accomplissements.

Mais ne nous trompons pas, c'est aussi celui qui guide la destinée de notre entreprise. Seules les plus aptes, les plus adaptées, celles qui auront réussi à mener à bien les changements nécessaires, celles qui auront mérité l'attention des actionnaires les plus exigeants, ces seules entreprises là auront le bonheur de survire. Et n'auront-ils pas honte nos collaborateurs, nos confrères, si à cause de leur négligence, de leur manque d'entrain à se battre pour notre groupe, les emplois de leurs collègues se trouvaient menacés à cause des meilleures performances de nos concurrents ?

Oui, je prétends et je signe que Polémos est le plus ancien et le plus fort, le plus à même de nous mener vers le meilleur de nous-mêmes. Il est celui qui amène chacun de nous à se rendre le plus utile à tous ceux qui l'entourent. C'est lui qui m'a menée, là où je suis aujourd'hui, c'est lui qui nous a menés là où nous sommes tous ensemble ce soir. Il sait distinguer les champions. Ceux dont nous avons besoin.

\section{F. ZANIAS}

« Ce ne devait pas être facile de commencer la première. Son discours était un peu sec, et pourtant elle semblait remporter l'assentiment général. En tout cas, tout le monde but en riant à la suite de son intervention. J'avais du travail pour remplir les coupes de tous les invités. Le seul qui ne riait pas était Filipo Zanias. C'est normal, il était représentant syndical, avant d'être nommé à la tête du comité d'entreprise. Lui n'était pas à son aise, quand c'était son tour de prendre la parole. Mais il s'en est malgré tout pas mal tiré.

F. Zanias : Je ne suis pas d'avis, Eve, qu'on nous ait proposé le sujet comme il convenait en nous proposant, sans distinguer, de célébrer Polémos. Il n'y aurait rien de plus louable que Polémos si tous les combats étaient les mêmes, mais je crois qu'il faut distinguer de quels combats nous sommes prêts à faire l'éloge.

Je crois que si nous devons célébrer Polémos comme il convient, il faut d'abord retenir la leçon des sports de combat. Que l'art martial doit être utilisé pour se défendre et non pas pour écraser.

Lorsqu'il s'agit de sauvegarder l'emploi, de défendre une région, s'il y a des injustices, s'il y a des profiteurs du système, s'il y a des camarades en difficulté, alors oui j'en appelle à Polémos car sans aucun doute c'est lui le plus fort. Ce contrat nous permettra d'éviter des licenciements et d'avoir plus de moyens pour nos actions sociales, alors je bois sans hésiter à ce Polémos qui nous permet de gagner.

Si le monde avance, si notre condition n'est plus celle de la révolution industrielle qu'analysait Marx, ce n'est pas grâce à l'amour du prochain, non, croyez-moi, c'est bien par la lutte et le combat de chaque jour. C'est le conflit, la contradiction qui fait progresser l'humanité, la lutte auprès de ceux qui en ont besoin, la lutte continue pour changer l'homme et le rendre meilleur, c'est à cela aussi que je lève mon verre. Et pourtant, je rêve chaque soir qu'un de ces soirs justement Polémos pourra se reposer. Je rêve que nous arrivions enfin à une société où les braves n'auront plus besoin de se battre 
encore, qu'ils pourront partager leur temps entre le travail, la création et leurs proches, où nous serons enfin devenus un grand tout, uni, qui ne fêtera Polémos que comme un ancien héro de l'histoire.

\section{STÉPHANE D'ARISTO}

«Il y eut comme un froid. Mais, si vous me permettez le jeu de mots, l'atmosphère n'était pas à la polémique. Tout le monde feint de rire, et devait reconnaître la finesse rhétorique de son discours. On passa vite au suivant. C'était à Stéphane d'Aristo de parler. Stéphane, c'était le directeur du marketing. Il avait passé plus de trente ans dans l'entreprise, autant dire une grande partie de sa vie. C'est une de ces personnes qui ont toujours l'air heureux de rencontrer un collègue dans les couloirs, et a toujours un mot gentil pour s'inquiéter de la santé ou de la famille de ses collègues.

S. d'Aristo : Si je devais vous initier aux pouvoirs de Polémos, il faudrait pour cela remonter aux origines de notre entreprise. Et toutes les belles et fortes entreprises ont débuté comme la nôtre. C'était au temps où ceux qui travaillaient à une même entreprise étaient plus soudés encore qu'une famille. Ils formaient un seul corps, sphérique, où chacun était à égale distance du centre : androgyne, solidaire, uni. Oui Filipo, nous avons connu dans le passé quelque chose qui ressemblait à ce que tu viens de nous décrire dans ton rêve de futur. Chacun était considéré avec une égale importance car chacun apportait sa part au pouvoir d'ensemble. Cet ensemble d'une seule pièce, androgyne et holiste, était admiré et respecté de tous. D'où que l'on regardait notre entreprise, on voyait une même belle harmonie, une égale puissance, et chacun de nous était si fier d'appartenir à ce tout admiré. Nous disions toujours 'nous' et nous aimions ces regards sur nous, tout de respect et de crainte.

Etait-ce sous l'influence de Zeus ou d'Hermès ?, était-ce l'œuvre d'un de nos concurrents jaloux ? mais notre entreprise fut découpée en deux centres, chacun faisant le compte de ses ventes et de ses charges. Et dès lors chaque centre n'a eu de cesse d'attirer sur lui tous ces regards qu'il avait pris l'habitude de recevoir et de savourer. Et cette quête a été si forte que chacun qui, dans le centre, contribuait à accroître l'admiration du centre était à son tour admiré et respecté de ses collègues, cité en exemple. Cette force d'ensemble qui nous rendait tous si fiers, chacun a voulu en être distingué comme le principal artisan. Car chacun voulait retrouver le plaisir de ces regards admiratifs, ce sentiment de force et d'importance, cette distinction qui rendait notre entreprise plus belle et enviable que les autres. On s'est tous mis à vouloir faire plus que les autres, pour avoir la plus grande part du regard. On s'est tous mis à espérer que les autres fassent moins bien que nous, un seul œil ne pouvait suffire à contenter tous les appétits. Les dieux se sont vengés de notre fierté à appartenir à ce tout androgyne, ils nous ont séparés en autant d'individus.

Pourtant depuis ce temps, chacun d'entre nous trouve une force inouïe dans ce besoin de reconnaissance. Pour ces yeux fiers et aimants, il donnerait tout son génie, toute sa force et son temps. Cette reconnaissance est ce qui le rend unique et lui livre un sentiment d'exister. Elle montre qu'il est digne et capable. Il éprouve la plus haute gratitude d'être ainsi l'objet d'un regard et d'une attention. La reconnaissance est son plus fort ressort, un ressort plus puissant encore que la solidarité du tout.

La puissance de Polémos, c'est qu'il nous pousse à donner le meilleur de nous-mêmes, il nous amène à aller tout au bout de nous-mêmes. Ce que nous voulions pour l'ensemble de l'entreprise, chacun le veut déjà pour lui-même, à sa place, à son niveau. Chacun veut compter pour les autres, plus que les autres, chacun montre ses comptes pour montrer qu'il faut compter avec lui, qu'on peut compter sur lui. Celui qui se bat pour avoir l'égard des regards, il veut être admirable pour les autres, car il veut être aimé.

Ce soir, nous buvons des coupes ensemble, mais dès demain matin il faudra individualiser et autonomiser la performance de nos collaborateurs. Pour que d'inconnus, ils deviennent reconnus. Parce que nous voulons des femmes et des hommes dont la plus grande crainte serait de retourner à leur origine androgyne.

\section{TEUSOK}

« Ils commençaient à rire très fort. Ils étaient habitués au discours plein d'images de M. d'Aristo. On sentait que chacun avait décidé de surpasser le précédent. Le directeur s'amusait à les voir rivaliser ainsi d'éloquence. Moi j'étais mal à l'aise, car je savais que si l'un glissait un mauvais mot, se laissait aller à une idée délicate, il risquerait gros. Je ne sais si vous pouvez comprendre ce 
mélange de détente et de tension dans l'atmosphère. Comme si même dans leurs rires, ils devaient rivaliser et calculer. J'ai même à un moment renversé quelques gouttes sur le costume d'un convive... Heureusement qu'ils étaient tous captivé par la cette joute oratoire.

En fait tout le monde attendait le discours de Teussok. Teussok, c'est le surnom que tout le monde lui donnait. Il avait un don inné pour se rendre agaçant. Il était le seul à oser contredire le directeur dans les réunions. Il posait toujours des questions, les questions qu'on n'attendait pas et qui soulevaient les points qui font mal comme on dit. Mais là, c'était soir de fête, ce n'était pas le jour à remuer les points délicats.

Teussok : Cher Stéphane, tu as parlé avec beaucoup d'éloquence, et je serais bien en peine de parler après toi avec une telle fougue. Je me suis laissé emporter par la force de séduction et de conviction de ton discours, mais je reste à la fin avec un petit étonnement. Veux-tu bien que nous en discutions?

$\mathrm{S}$ : Je serais ravi d'éclaircir ce que je n'ai pas su parfaitement exprimer.

- Si nous faisons la guerre c'est bien pour ou contre quelque chose qui nous est important ?

$\mathrm{S}$ : Assurément.

- De sorte que s'il n'y avait pas la guerre, nous ne ferions pas les choses que celle-ci nous conduit à entreprendre.

$\mathrm{S}:$ Je ne l'aurais pas dit plus avec plus de perspicacité.

- Mais aller au meilleur de soi-même n'est-il pas ce que nous voudrions tous faire et d'autant mieux que nous ne serions pas accaparés par les joutes de Polémos?

$\mathrm{S}:$ Absolument.

- Donc ce que Polémos nous enjoint de faire c'est autre chose que de nous emmener au meilleur de nous-mêmes.

$\mathrm{S}:$ Je suis forcé de le reconnaître.

- C'est donc que la lutte et le conflit nous amènent à développer soit le pire de nous-mêmes, soit nous amènent vers autre chose que chose que nous-mêmes, ou quelque chose que nous ne développerions pas de nous-mêmes.

$\mathrm{S}:$ Jusque là je te suivrais volontiers.

- Je laisserai à d'autres le soin de développer le pire de nous-mêmes et voudrais te conter une rencontre qui m'a fait comprendre que Polémos nous fait tant différer de nous-mêmes que je ne sais plus ce que soi-même peut bien signifier. Je voudrais donc vous rapporter le discours de cette femme, Moneytime, qui m'a enseigné sur la guerre bien plus que nous tous ici réunis nous pourrions bien parvenir à dire.

J'avais tenu un discours bien proche de celui de Stéphane quand Moneytime se moqua de moi, arguant que la lutte pour la survie et la compétition ne saurait nous faire développer le meilleur de nous-mêmes.

Mais alors Moneytime, lui ai-je dit, quel est donc ce démon ? Et d'où vient-il ? Qui peut bien être son père et qui est sa mère ?

C'est une longue histoire que je vais essayer de te résumer, me répondit Moneytime. Le jour où naquit Polémos, les Dieux faisaient un grand festin. Et parmi les convives, Position fêtait tous ses territoires et les gloires qu'elle en tirait. Mais du fait de l'abondance de nectar qu'elle avait bu, elle s'était endormie ivre sur une banquette. Là survint dans un de ces brefs passages desquels il était coutumier, Mouvement qui prit un instant de repos dans le giron de Position. Quand elle se réveilla, Mouvement était déjà bien loin, mais elle avait gagné une nouvelle possession dans un nouveau-né qu'elle nomma Polémos. De son père Mouvement, il a hérité d'une nature toute instable. Il est toujours animé par les fuites, les coups, les tours ; il se repositionne chaque fois, s'éloigne quand on veut l'attraper, réapparaît quand on croyait l'avoir banni. Il n'appartient à personne, ne dort que d'un œil et semble dépérir chaque fois qu'on veut le maintenir en place. Mais de sa mère, il est toujours à vouloir gagner de nouvelles positions et possessions, il veut de l'ordre et de la discipline dans son empire, il attribue des places et des classes, il bloque et étouffe les lieux assiégés bien qu'il ne semble jamais se suffire de ce sur quoi il règne.

- Je ne saurai pas mieux décrire la double nature de Polémos! 
M : Maintenant que nous savons d'où vient Polémos, peux-tu me dire ce que toujours il cherche?

- C'est justement ce que j'allais te demander.

$\mathrm{M}$ : Je vais te parler de ceux qui dirigent, que ce soit les grandes institutions ou les entreprises. Il y a chez chacun de ceux qui suivent les traces de Polémos le désir de réaliser de grands projets, que ce soit pour la société, pour le groupe qu'ils dirigent, ou pour eux-mêmes. Mais ces projets ne se font pas par eux seuls, aussi cherchent-ils la plus grande puissance.

- Ils veulent du pouvoir, à n'en pas douter.

M : Mais il y a le pouvoir qui vient de la force et de la crainte, celui qui vient de l'institution et des positions et puis celui qui est gagné par l'adhésion. Aussi celui qui aime véritablement Polémos ne cherche-t-il pas de la reconnaissance. Il désire tantôt être craint, tantôt se rendre invisible pour utiliser la force des processus et des institutions et d'autres fois encore être justement l'œil, non celui qui reçoit mais celui qui distribue la reconnaissance.

- Alors initie-moi à l'art de Polémos.

M : Il te faut d'abord apprendre la contemplation des idées en général et savoir quel est pour toi le juste projet. Il te faut ensuite atteindre à l'intelligence de l'économie et de la politique afin de connaître l'état des forces en général. Puis l'art de traduire ton projet en beaux discours et justes raisonnements. Quand tu as su faire cela, alors tu peux peser pour orienter les interprétations des parties-prenantes et fixer les règles. Lorsque tu seras passé maître dans l'art des règles et des significations, il te faudra apprendre à les imposer sur les corps et les représentations. Il te faudra alors aimer les corps au travail car c'est sur eux que finalement s'imposera ta puissance.

- Je ne peux que reconnaître ce qui devrait être enseigné dans les meilleures formations !

$\mathrm{M}$ : C'est en effet la droite méthode pour accéder de soi-même aux choses du conflit ou pour y être conduit par un coach. C'est en prenant comme point de départ la beauté des idées et des projets en général puis de descendre sans arrêt comme au moyen d'échelons : partant d'un beau projet descendre dans les discours et les raisonnements ; puis partant des discours et raisonnements descendre vers les règles et les interprétations ; puis partant de ces dernières s'imposer dans les têtes et dans les mouvements des corps ; enfin terminer dans l'appropriation par chaque corps des gestes, des sensations et des rythmes qui s'inscrivent dans le projet désiré.

- J'inscris déjà cette méthode dans un séminaire de cinq journées.

M : Quoi de plus beau dans l'existence que d'amener tous ces autres dans l'accomplissement de son projet. Aussi est-ce mon opinion, je le déclare, une obligation pour toute personne de vénérer Polémos et il n'est pour moi de plus grande réussite que dans cette alignement, ce concours, des projets et des beaux corps au travail.

\section{ALCIDE BIAD}

« Teussok n'avait pas fini son discours qu'il fut interrompu brutalement. On entendit de grands cris venant du hall d'entrée, où les insultes se mêlaient à des cris de douleur. Alerté, le directeur voulut montrer qu'il n'avait pas peur et alla s'enquérir de ce qu'il en retournait. Il reconnut alors Alcide, Alcide Biad, son ancien collaborateur, non rasé, dans des habits de loques et plein d'odeurs d'alcool. Il pria qu'on le laisse entrer et qu'il puisse avoir comme les autres une coupe de champagne. Il prononça même quelques mots de bienvenue. Quand Alcide fut mis au courant de la règle du jeu pour cette soirée de cocktail, il demanda la parole.

A. Biad : J'ai honte de paraitre ainsi devant vous, alors que vous aviez l'habitude de me voir toujours impeccable et dans des costumes des meilleures factures. Et je ne sais si je saurai encore correctement parler ce soir. Je suis ivre, tout autant d'alcool que de colère et d'amour, ivre de la joie d'être de nouveau ici. J'imagine facilement que chacun ici aura parlé avec un juste détachement de la guerre, mais c'est d'amour que je viens moi vous parler.

Quand je suis arrivé dans cette entreprise, j'entrepris d'être le plus près possible de vous, car je savais qu'en étant près de vous, en écoutant vos paroles et regardant vos actions, $j$ 'allais apprendre à devenir meilleur. J'aimais vos projets, je savourais vos discours et je crois que je montrais un zèle impeccable pour mériter votre proximité. J'ai changé mes façons de parler et de me vêtir, je restais tard le soir pour que tout ce qui m'était confié reçût un traitement exemplaire et digne de notre groupe. J'emmenais toujours ensuite quelques dossiers avec moi pour préparer le lendemain. 
Je m'aperçus à peine que mes amis s'étaient détournés de moi car je n'avais plus de temps pour les voir. Le jour où ma femme, qui m'avait au début tant soutenu, est partie avec les enfants, je pris cela presque comme une délivrance car j'avais ainsi plus de temps encore pour me consacrer à mes ouvrages.

Arrêtez-moi si je mens, mais rappelez-vous comme j'aimais être fouetté par la chaîne de valeur, quand il fallait se sacrifier, se scarifier pour les résultats. J'aimais le stress et la pression, j'aimais les conflits difficiles avec ceux qu'il fallait presser plus encore - la fatigue dans mon corps avait le goût du travail bien fait. Je ressentais dans ma chair la dureté des chiffres, et je me récitais pour me consoler notre mission au format powerpoint. Je me sentais être homme, je savourais les projets de changement au goût de cuir ; la discipline implacable, les logiques irréfutables, les mesures comptables avaient la dureté du fer mais produisaient comme une musique sublime. Et le chant des projets était si beau... J'étais à n'en pas douter devenu amoureux de mon entreprise et de ses chefs, amoureux de ceux qui m'avaient kidnappés à ma vie et à mes proches - un syndrome de Stockholm d'autant plus dangereux qu'il était partagé par beaucoup d'entre nous.

Oui les idées et les projets étaient inscrits profonds au-dedans de mon corps, si bien que je ne savais plus où finissait l'entreprise, et où commençait ma vie. Et je le dis devant vous comme témoins, et personne ici n'osera prétendre le contraire, j'étais heureux. Je me sentais important, je me sentais appartenir à plus grand que moi et chaque jour se présentait comme un nouvel exploit à accomplir.

Mais les projets devenaient chaque fois plus exigeants, et les comptes plus rudes chaque mois, si bien qu'un jour comme un coup sublime, comme un résultat proche de la perfection, je décidai de délocaliser ma division et de démissionner pour des comptes montrant un progrès inattendu.

Le lendemain de ce jour de gloire je n'avais plus rien, je n'étais plus rien ; et l'absence de projets ne tarda à s'inscrire dans mon corps, jusqu'à ce qu'il devienne cet objet de dégoût autant pour vous que pour moi-même. Mais quoi qu'il soit devenu aujourd'hui, je n'ai jamais cessé, de tout mon corps et tout mon cœur, d'aimer cette entreprise et ceux qui l'élèvent à sa plus splendide puissance.

Voilà ce que je suis venu vous dire ce soir. Et ne vous y trompez pas, mon cœur est plein de gratitude. C'est jour de fête que d'être de nouveau parmi vous. Donnez-moi encore une coupe de champagne, que je porte un toast aux beaux projets et à la contemplation des idées, un toast à l'art de la guerre dans les affaires et à l'hoplite, ou plutôt au samouraï que vous m'avez permis de devenir.

\section{$\ll \mathbf{L} »$}

« Il fallait voir combien il était heureux ce soir là. Comme un toxico qui, après une longue cure, se voit donner une nouvelle dose. Il but beaucoup, beaucoup de champagne. Je ne sais pas s'il me reconnaissait quand je le servais. Depuis ce soir là, on ne l'a plus revu, c'est un mystère. Personne n'a reçu de consigne, mais je n'ai pas entendu un seul des invités dire un mot au sujet de ce cocktail.

Dix ans déjà. Mais vous allez en parler n'est-ce pas ? Vous écrirez un livre là-dessus. Il faut que les gens sachent. Vous donnerez mon nom n'est-ce pas ? Vous direz que c'est moi qui étais là, moi la première qui ai osé en parler. Je me moque bien des conséquences maintenant. Vous comprenez, j'ai toujours été la gentille fille, celle qui fait passer les autres devant et encore dit merci lorsqu'elle s'est fait marcher sur les pieds.

J'ai des informations importantes à vous révéler. Vous savez, l'assistante du directeur que j'ai remplacée ce soir là, auparavant elle était dans mon service. On travaillait dès le départ sur le projet du contrat qui était fêté. Mais lors d'une réunion, elle présenta les choses comme si c'était elle qui avait tout fait. Elle racontait dans les couloirs des horreurs sur mon compte. Et elle avait l'oreille de la direction. Il faut dire qu'elle était mignonne cette garce. Je n'ai pas compris alors ce qui se passait. Un jour c'est elle qu'on chargea du dossier, et moi qui devais travailler pour elle ! Elle ne me laissa plus aller en réunion, se débrouilla même pour que mon bureau fût transféré dans l'annexe afin que je ne voie qu'exceptionnellement tous les dirigeants. Après s'être attiré toute ma gloire, elle avait réussi à me neutraliser. Mais c'est moi, il faut qu'on le sache, qui ai fait réussir ce contrat ! Je voudrais que vous écriviez un livre là-dessus, où tout sera révélé. Chaque détail. Maintenant je peux parler, maintenant elle ne me nuira plus. C'est comme ce soir là ; le soir du cocktail - c'était mon unique victoire - j'avais réussi à lui faire croire qu'elle aurait des informations inédites sur une autre de ses rivales si elle allait dans la filiale d'Helsinki. J'ai réussi à l'éloigner, et c'est moi qui servais le champagne au directeur... 
Ecrivez ce livre sur ce cocktail, sur toute cette histoire, sur mon histoire. Je veux sortir de l'ombre, je veux que le dirigeants enfin reconnaissent qui j'ai été et tout ce que j'ai fait pour notre entreprise. Dix ans que j'essaie de la détrôner, de reprendre la place qui m'appartient, que je mérite. Mais jusqu'ici elle était trop forte. Alors ce livre, ce sera ma vengeance. Je veux être dévoilée, je veux être racontée, car sinon je ne sais plus qui je suis, pour qui j'existerais ? Un livre s'il vous plaît, un livre, je vous y aiderai... »

Je fis un sourire qu'elle interpréta comme un accord. Après tout, $\mathrm{j}$ 'aurais sans doute encore besoin des ses témoignages. Elle était mes données de terrain, la preuve qu'il me fallait pour valider mon enquête. Il fallait qu'elle collabore à mon projet.

Mais un livre, ça non, il n'en est pas question. Un livre n'est pas assez apprécié lors des évaluations. Je veux être le premier de ma discipline. Et seuls les quelques meilleurs auront une place. Je veux être connu et reconnu. J'ai de beaux projets de publication et mon corps apprend la dure discipline pour gagner cette place. Ces heures d'écriture académique, ces nuits à travailler, ces bouffées de stress avant un rendez-vous, ces réunions pour les yeux de la direction. Je ne suis plus le même depuis quelque temps. J'ai changé. Mes amis me le disent, même si je ne les vois plus beaucoup. Mais j'ai le sentiment de devenir meilleur, plus apte dans ce monde hostile en tout cas. Dans ce monde de la guerre de tous contre tous.

Si elle m'aide, c'est pour elle. C'est parce que c'est sa dernière cartouche. Un livre attirerait encore l'attention des dirigeants et elle aurait du pouvoir sur eux par ses révélations. Mais ce sera une série d'articles dans des revues que ces dirigeants ne liront pas. Il faut que je pense à moi. Je ne veux pas devenir comme cette femme. Je veux être de ceux qui boivent le champagne aux cocktails, non ceux qui le servent. 